\title{
Distance perception in an open water environment: Analysis of individual differences
}

\author{
Chris Button $^{1} \cdot$ Matthew Schofield $^{2} \cdot$ James Croft $^{3}$
}

Published online: 29 December 2015

(C) The Psychonomic Society, Inc. 2015

\begin{abstract}
We investigated whether distance estimation accuracy over open water is influenced by the viewing direction of the observer. Twenty-two healthy students ( 9 male, 13 female) made 10 distance estimates ranging between 50 and $950 \mathrm{~m}$ actual distance in 2 viewing conditions: (1) from shore to boat and (2) from boat to shore. There were no consistent differences in estimation accuracy between viewing directions. The group data revealed a general tendency to underestimate actual distances (74\%), but there was considerable interindividual variance (mean error of $74 \% \pm 27 \%$, range $=31 \%$ to $145 \%$ ). A multilevel regression model of estimate accuracy suggests there were three subgroups of participants. One subgroup $(N$ $=4,18 \%$ ) were consistent underestimaters, regardless of distance, whereas another subgroup $(N=5,23 \%)$ consistently overestimated. However, the majority $(N=13,59 \%)$ tended to underestimate at shorter distances (less than $400 \mathrm{~m}$ ) and then overestimate at longer distances. These findings have important implications in survival situations in open water where an individual may need to judge an estimated distance against their perceived swimming capacity in order to self-rescue.
\end{abstract}

Chris Button

chris.button@otago.ac.nz

1 School of Physical Education, Sport and Exercise Sciences, University of Otago, Dunedin, New Zealand

2 Department of Mathematics and Statistics, University of Otago, Dunedin, New Zealand

3 Centre for Exercise and Sports Science Research, Edith Cowan University, Perth, WA, Australia
Keywords 3D perception $\cdot$ Space perception $\cdot$ Perception and action $\cdot$ Scene perception

The visual system has access to multiple types of information for distance perception, such as texture compression, perspective, retinal size, and vergence (Gibson, 1979). Yet despite the typical abundance of such cues, numerous factors, including perceptual biases, viewing perspective, and degradation of cues, can result in humans making distance estimation errors. In some extreme situations (e.g., military, search and rescue), distance estimation errors may prove to be fatal. In this experiment, we considered how people make distance estimations over open water. We also investigated how distance estimation is influenced by the viewing direction of the observer (i.e., looking from water back to shore vs. from shore out over water).

Estimating the distance to objects in the sky or over open water presents humans with particular difficulties because foreground and background texture gradients and contrasts are often unreliable or absent. Indeed, distance perception in situations of cue reduction is challenging because the relationship between the angular extent of objects and perceived size need not necessarily be consistent with the perceived distance (Brenner \& Van Damme, 1991; Gogel, 1971). For example, a general tendency for humans to underestimate overland distances up to approximately $25 \mathrm{~m}$ was found by Ross (1967) and Franklin, Ross, and Weltman (1970). In relation to larger distances, Fine and Kobrick (1983) asked soldiers to estimate the distance of a military truck placed between 600 and 1,550 $\mathrm{m}$ away from them on a large, flat, open grassy area. The average data indicated reasonably close approximation of distance; however, close inspection of the data revealed two distinct subgroups of participants. One subgroup (inexperienced soldiers) consistently underestimated overland distance, 
whereas the other subgroup (experienced military personnel) were more likely to overestimate. Interestingly, it seems that through experience people somehow learn to compensate for the tendency to underestimate (Fine \& Kobrick, 1983). Daum and Hecht (2009) also examined distance perception using a field study of an outdoor, overland vista in which relatively few cues were present. Participants were required to estimate the distance to square wooden boards of different sizes $\left(0.4 \mathrm{~m}^{2}\right.$ and $1 \mathrm{~m}^{2}$ ) placed 27 to $217 \mathrm{~m}$ away within vista space. Overall, there was a tendency to underestimate $(68 \%$ of true distance) when the viewed object was less than approximately $100 \mathrm{~m}$ away and overestimate distances (255\%) above this threshold, suggesting a nonuniform vista space in terms of the accuracy of distance perception. Unlike Fine and Kobrick's (1983) study, no analysis of individual data was offered by Daum and Hecht (2009), so it is unclear whether this interpretation applies consistently at an individual level.

Despite being important in various life-threatening situations, there are few published studies pertinent to the issue of distance estimation over open water. In one exception, Baird and Burkhart (2000) asked passengers and sailors to estimate distances from a boat to a humpback whale up to $600 \mathrm{~m}$ away in the ocean. Overall, observers were reasonably accurate but quite variable (accuracy $90 \% \pm 68 \%$ ), and different subgroups of participants tended to either underestimate or overestimate the distance. Interestingly, experienced observers (i.e., sailors) showed a similar level of accuracy but were much less variable (accuracy $89 \% \pm 22 \%$ ) in their decisions than the less experienced passengers. To our knowledge there is only one other published study that has considered the influence of viewing direction on distance estimation over open water (Higashiyama \& Shimono, 1994). In this study, one set of observers judged distances (1.1-15.3 km) from a viewing platform to other landbased objects on nearby islands and were shown to typically overestimate the true distances. In contrast, another set of observers viewed the landmarks from a boat over similar distances and were on average quite accurate. It is possible that distance estimation is worse when looking out toward open water (than looking back to shore) due to a relative lack of familiar objects in the viewed environment and an absence of background texture. In the present study, we wished to verify whether the between-group viewing direction differences found by Higashiyama and Shimono (1994) would be replicated with a within-group experimental design.

To summarize, estimating distance over open water is likely difficult due to the ambiguous information available to inform judgements. The limited research conducted to date is equivocal in terms of the level of accuracy one might expect and also whether over- or underestimates are more likely across open water. Based on previous work (Higashiyama \& Shimono, 1994), we predicted that distance estimates would be most accurate and reliable when made from a boat looking back to land rather than vice versa.

\section{Method}

Participants were recruited from a cohort of 32 physical education university students. Individuals with prior experience of life-saving or open-water swimming were excluded from participating in the study. From the original cohort, 22 students volunteered to participate and signed informed consent prior to testing. They were instructed about the nature of the testing but were not told about the distances that they would be required to estimate. The testing procedures, detailed below, conformed to the ethical requirements of the participating institution. The participants were given no prior distance estimation training or familiarization with the testing environment.

Testing took place in a channel of a harbor waterway on two different days of comparable weather conditions (i.e., fine, clear, light breeze, and calm water). It was necessary to test over 2 days due to limitations in the capacity of the boat to carry no more than eight people (including driver and experimenter) at any one time. Before testing began, the boat started at the shore close to the participants so that they were aware of its size $(6.5 \mathrm{~m}$ long $\times 3.5 \mathrm{~m}$ wide $\times 3 \mathrm{~m}$ above water height). The participants made distance estimations from two different viewing perspectives (from shore out to the boat and from the boat back to shore). From each viewing perspective, participants made 10 distance estimations (in units of meters) from actual distances ranging between 50 and $950 \mathrm{~m}$. The distances were selected in four random orders by the experimenter. After the 10 estimates were completed (approximately 20 minutes), the two subgroups then swapped viewing positions and repeated the procedure.

On each testing day, approximately half of the participants first made the distance estimations from the shore to the boat. At the same time, the rest of the participants standing on board the boat made the estimates back to the shore. When viewing from the shore there were no other objects (e.g., boats) within close vicinity, apart from the other participants making estimates on the boat, which participants might have used to extract familiar size cues (see Fig. 1a). In contrast when viewing from the boat, there were several familiar objects (e.g., participants, car, houses, trees, launching jetty) on the shore that may have helped participants to make their estimates (see Fig. 1b). In between estimations, each set of participants turned around so that they were unable to see either the boat or shore to extract distance information from the boat's movements (see Daum \& Hecht, 2009). The participants were not allowed to talk during the distance estimates, and they each had to write down their estimations on paper to prevent them from influencing each other. Participants were not given feedback regarding the accuracy of each estimate. The actual distances were measured using a prism mounted on a rod fixed to the boat and a surveying theodolite (TCR 307, Leica Geosystems AG, Switzerland) secured to a tripod set on the shore. The theodolite was equipped with an electronic distance 


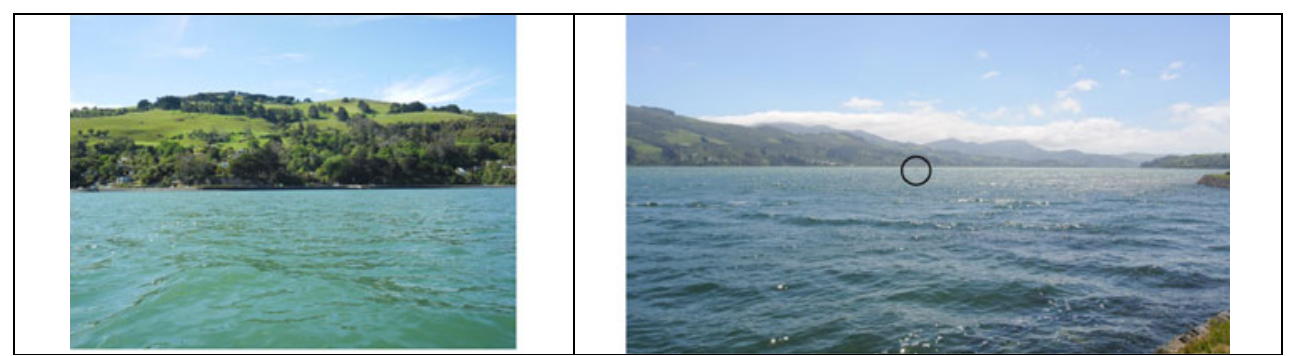

Fig. 1 Example photographs of the experimental environments taken from a boat $400 \mathrm{~m}$ from the shore (a, left) and from the shore looking out to the boat $(\mathrm{b}$, right). The location of the boat on the horizon is marked

measurement device and is accurate to within $2 \mathrm{~mm}$. While on board the boat, participants wore a life jacket for safety reasons.

Utilizing the estimated and actual distances, the estimation error expressed as a percentage of actual distance and interindividual variability (i.e., $S D$ of estimated error) were calculated. Fine and Kobrick (1983) council wisely that data analysis procedures should be sensitive to the individual differences that are likely to exist in distance estimation tasks. Hence, we used a regression model describing the estimated distances in terms of the actual distances with individual-specific intercept and slope terms (for more details of the models, see Appendix A.). As well as allowing for the individual-specific intercept and slope terms to differ between the two perspectives, the intercept was modeled in terms of three covariates: (i) order of condition (boat or shore perspective first); (ii) prior estimating experience (from the other condition); and (iii) influence of the previous estimate. These individual based models are examples of multilevel or hierarchical models (Gelman \& Hill, 2006), with Bayesian inference used for model fitting (Gelman et al., 2014).

\section{Results}

When the group estimation errors were averaged across testing days and distances, a similar level of underestimation was found from both viewing directions (i.e., $74 \% \pm 22.3 \%$ from the boat, $74 \% \pm 32.3 \%$ from the shore; see Table 1 ). While the accuracy is similar between viewing directions, at most distances the dispersion of estimates is largest in the shore to boat condition (see the gray data points in Fig. 2). There was some indication that the four randomized distance orders may have a stronger influence upon estimation error than viewing direction. This possibility was investigated further in the individual analysis.

The posterior probability of making estimation errors are presented in Fig. 3. The order of participants is presented such that individuals toward the top of Fig. 3 were more likely to overestimate distance while individuals placed toward the bottom were more likely to underestimate. It is notable that some individuals (A-D, 4 cases) appear likely to overestimate distance quite consistently while some individuals (R-V, 5 cases) show low probabilities of overestimates across the distances examined. The by a black circle to aid identification for readers. No such artificial cues were available to participants during testing

remaining participants (E-Q, 13 cases) typically show a low probability of overestimating shorter distances and this probability tends to increase with distance. As the group averages indicated, there was also little evidence at the individual level that viewing direction consistently influenced estimation error.

We wished to explore to what extent the variation within and across individual responses can be explained by other variables of interest. Hence, the intercept and slope terms were first modeled in terms of viewing direction, confirming that the extent and rate of estimation error did not change as a function of direction with the $95 \%$ credible intervals crossing zero (see Fig. 4). An additional model was created that allowed the individual-specific intercept term in the regression to depend upon covariates (irrespective of the actual distance). Extending the original model revealed no evidence that the order of viewing directions presented to participants (i.e., from boat or shore first) influenced estimation error. The two covariates that did explain variance in estimation error were prior estimating experience and the influence of the previous estimate on the current estimate. It is notable that some of the most accurate sets of responses came from participants making their second set of estimates (see shaded cells in Table 1). There is also evidence that underestimating in the previous trial is associated with a decreased estimate in the current trial. Likewise, overestimating in the previous trial is associated with an increased estimate in the current trial.

\section{Discussion}

Overall, participants estimated distances over water relatively accurately, with a general tendency to underestimate actual distance (mean error of $74 \% \pm 27 \%$ ). Viewing direction appeared to have little influence upon distance estimation capability, although there was a little more individual variation when estimating from the shore (i.e., shore to boat $S D=$ $32 \%$ vs. boat to shore $S D=22 \%$; see Fig. 2). Given the lack of contextual information available across open water to guide estimates, it was perhaps surprising that the accuracy was so high. However, comparing our findings to those of Baird and Burkhart (2000), whose participants were estimating distances 
Table 1 Summary table of mean estimation error ( $\%$ of actual distance) and interindividual variability $( \pm S D)$. The unshaded cells were calculated from participants making their first set of estimates. The shaded cells were calculated from participants making their second set of estimates. The arrows indicate the order of perspectives presented to the four subgroups of participants

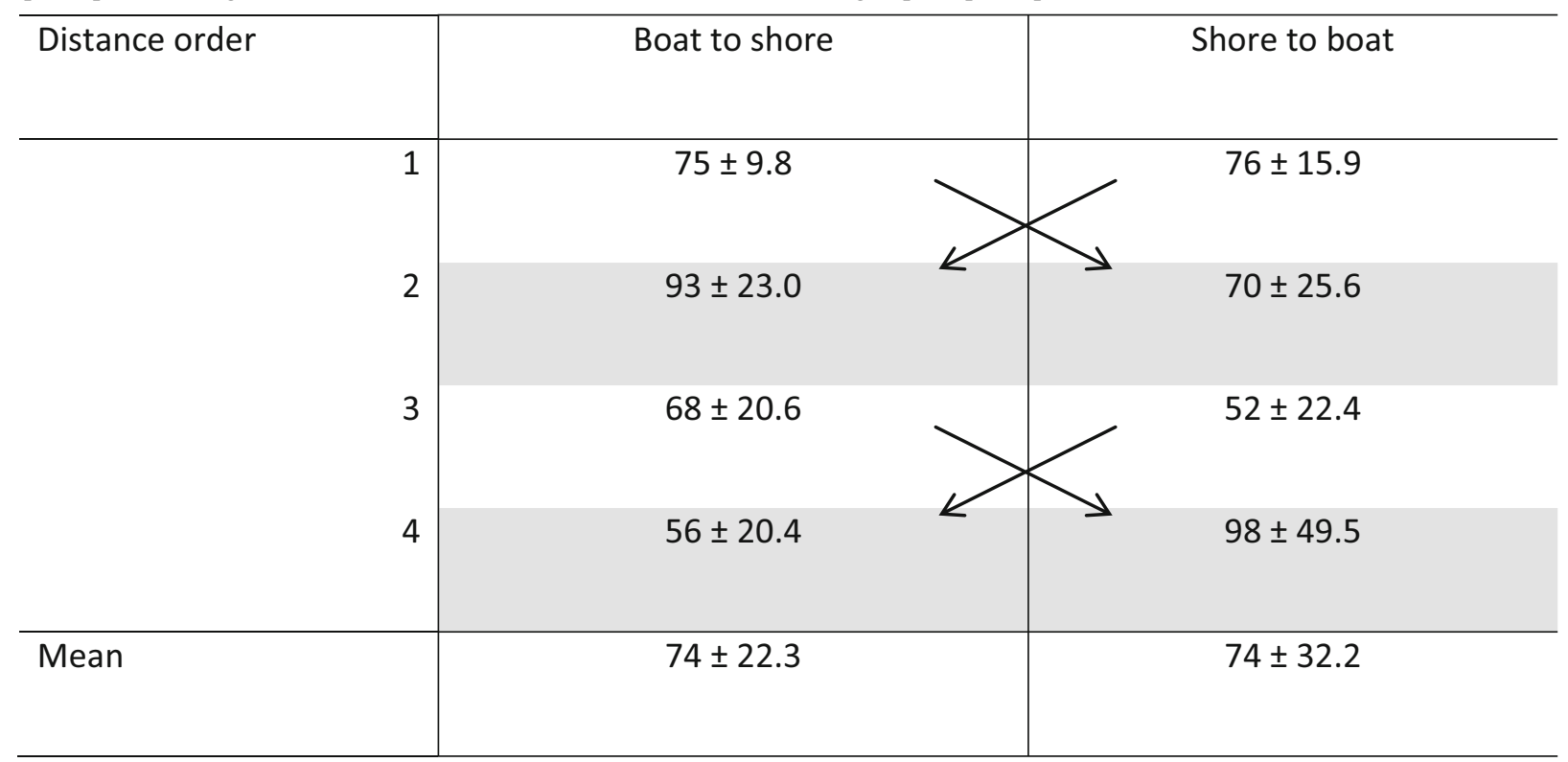

from a boat to a humpback whale, a similar level of accuracy is apparent (i.e., $89 \% \pm 60 \%$ ). Both sets of data suggest that estimates made over open water are subject to similar biases as those made over an open land vista (Daum \& Hecht, 2009).
Higashiyama and Shimono (1994) found that a group looking away from shore out to other islands up to $15.3 \mathrm{~km}$ away overestimated distances. In contrast, our data (albeit over reduced distances up to $900 \mathrm{~m}$ ) indicate that estimates

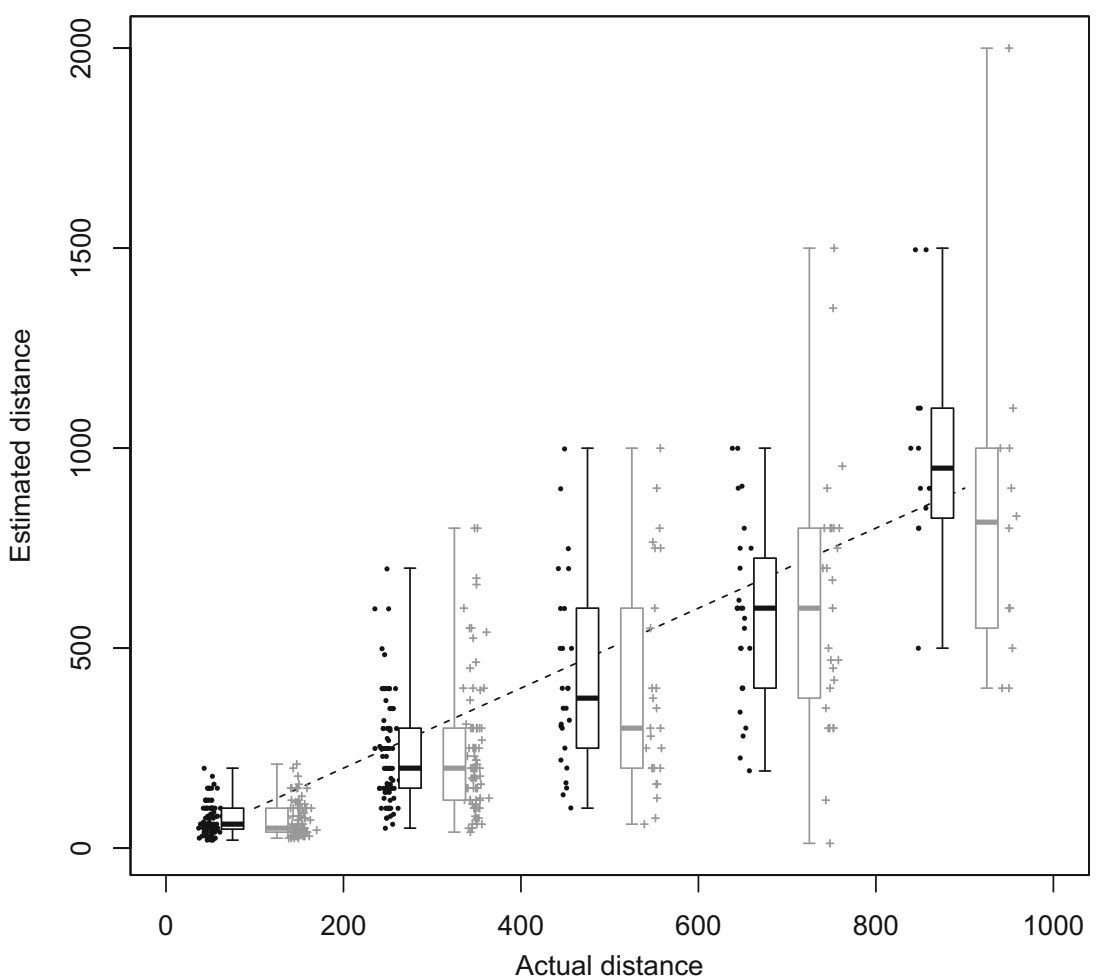

Fig. 2 Mean distance estimates and standard error represented as box and whiskers plots summarized into distance bins (i.e., 100, 300, 500, 700 , and $900 \mathrm{~m}$ ). Individual data points are overlaid next to the boxes to help visualize the dispersion of data. The dark font is the boat-to-shore data. The gray font is the shore-to-boat data 


\section{Overestimation}
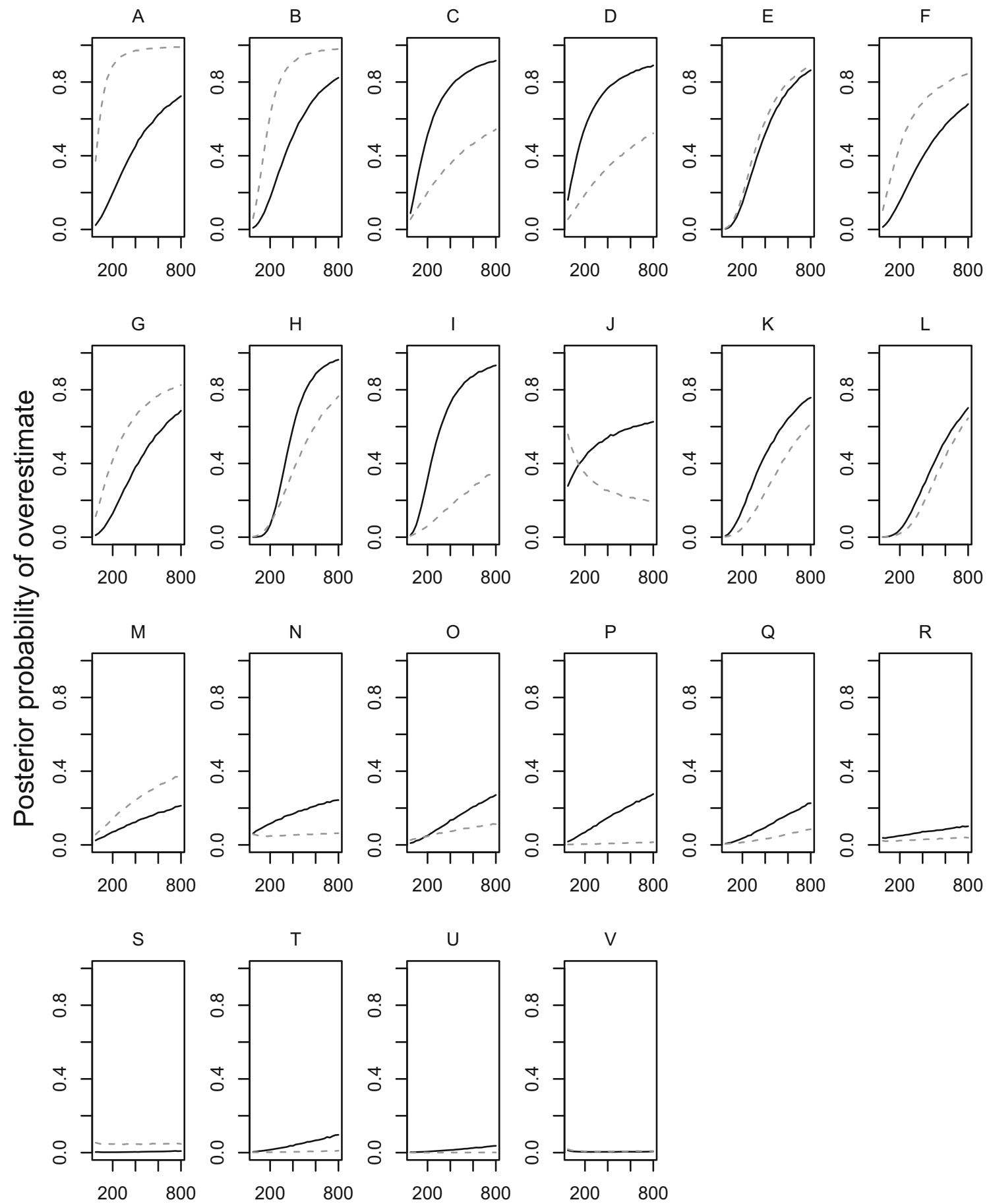

Actual distance $(\mathrm{m})$

Fig. 3 Posterior probability of overestimating the distance by individual. Each fitted model plotted is a separate individual. The solid line denotes the boat-to-shore condition. The dotted line denotes the shore-to-boat condition

are as accurate, if not as reliable, when made from the shore as they were from a boat looking back to shore. Hence, individuals presumably adopt strategies that compensate for the lack of background contrast when making estimates to objects of familiar size in open water. Instead it seemed that the influence of the previous estimate made or prior estimating experience were stronger influences on estimation accuracy than viewing direction (see Fig. 4). Individuals were more likely to overestimate if they had previously overestimated and were more accurate having completed one set of estimations (regardless 


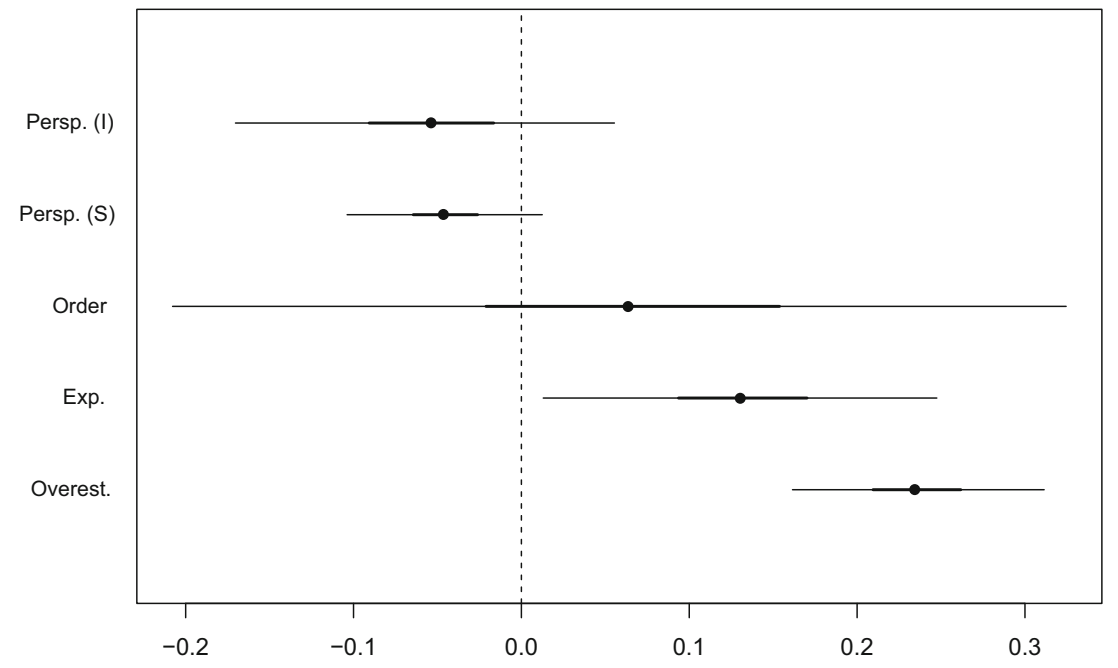

Fig. 4 Caterpillar plot of $95 \%$ credible intervals for multiple parameters associated with the posterior probability of overestimating. Persp. (Intercept) and Persp. (Slope) represent models where these individual regression parameters were allowed to vary by viewing perspective.

of direction). As participants were not provided with any feedback about accuracy during the experiment, one can assume that prior history of estimating was an influential cue used to inform subsequent judgements.

When one considers the group data alone, the individual differences in estimation response are hidden (Fine \& Kobrick, 1983); hence, we created an individual-based multilevel model in order to better describe the differences. As such, it became apparent that some individuals (IDs: A-D, 18\%) consistently overestimated regardless of condition or distance, while others (IDs: R-V, 23\%) overestimated most of the time. The remaining participants (IDs: E-R, 59\%) underestimated at smaller distances and then became more likely to overestimate at larger distances (see Fig. 3). According to Daum and Hecht (2009), human perception of vista space overland is not uniformly (in)accurate across distances. At short distances (i.e., less than approximately $100 \mathrm{~m}$ ) humans typically underestimate distance; at longer distances they typically overestimate with increasing error and variability. Data from a large proportion of participants in the present study $(N=13,59 \%)$ confirmed these findings. The threshold at which people tend to switch from underestimating to overestimating seems to be higher over open water than reported over land (75-100 m) by Daum and Hecht (2009), as indicated by Fig. 3, in which the probability of overestimates was generally low until at least $400 \mathrm{~m}$. It is possible that the larger target object (boat) and greater range of distances used in our study in comparison to Daum and Hecht's study $\left(0.4 \mathrm{~m}^{2}\right.$ and $1 \mathrm{~m}^{2}$ wooden boards from 27 to $217 \mathrm{~m}$ ) may have shifted this perceptual threshold.

Another smaller subgroup of participants $(N=5$ participants R-V, 23\%) responded quite differently to the other subgroups by consistently underestimating regardless of distance and viewing perspective. One possible explanation for the
Condition order, experience, and previous overest(imate) represent three covariates added to an extended model. The values on the $x$-axis reflect the effect size for each of the predictor variables. Full descriptions of the model are given in Appendix A

underestimation phenomenon is that of perceptual scale expansion offered by Durgin and Li (2011). Scale expansion occurs because the perceived gaze declination between an observer and viewed object is magnified in cortical processing. Underestimates of distance seem to be more common in virtual environments (Ziemer, Plumert, Cremer, \& Kearney, 2009). It is conceivable that the impoverished information in open-water environments mimic to some extent those encountered in virtual worlds, leading to a tendency for some people to compress distance. While there was individual variation in responses, each individual appeared to be quite consistent in their own estimating strategy. The potential mechanisms underlying this interesting feature of the data require further investigation in future work.

In terms of potential limitations to the study, it is possible that participants used additional cues to inform their estimates that were not controlled in the experimental design. For example, while traversing distances in the boat, participants may have used the speed or direction of the boat to help inform their estimates. However, this seems unlikely given the lack of differences between perspectives even at the individual level. Another factor that could have influenced distance estimation is familiarity with the location. Individual differences are to be expected in distance estimation studies if participants can use experience and familiarity with the scenery to help inform their judgments (Willey \& Jackson, 2014). Although a remote channel of an open harbor was used for testing, each individual's relative familiarity with the area was not controlled for and represents a potential limitation. When making the estimates, participants could also view the other group clearly (either on shore or on the boat), which might have helped by providing familiar objects to contrast against. Delimitations like the familiarity of the boat's size to 
the participants and also the restricted range of distances $(0-950 \mathrm{~m})$ preclude us from drawing detailed conclusions about the perceptual strategies used from the different viewing perspectives in the present study.

In conclusion, participants were quite consistent and accurate in their ability to estimate distance over open water, albeit with individual variation. There were no differences in estimation accuracy when viewing from shore to boat or from boat to shore across the relatively narrow range of distances examined (under $1 \mathrm{~km}$ ). The majority of individuals tended to underestimate smaller distances and then overestimate larger distances. However, some individuals consistently underestimated distance regardless of distance or viewing perspective, whereas some individuals were consistent "overestimaters." While numerous potential factors likely contribute to these individual biases, the prior influence of making estimates and relative experience contribute most to estimation accuracy. When making distance estimates over open water, it is likely that people will underestimate the actual distance (particularly less than $400 \mathrm{~m}$ ). This finding has important implications in survival situations in open water where an individual may need to judge an estimated distance against their perceived swimming capacity to effect self-rescue.

Acknowledgments We acknowledge the assistance of Miss Milou Brand (research student) and Mr. Neil George (boat driver) in collecting the data. We also acknowledge the students who volunteered to participate in the study.

\section{Appendix A}

\section{Individual-based regression}

The data are denoted by $y_{i j h}$, the $\log$ of the distance stated by individual $\mathrm{i}=1, \ldots, \mathrm{m}=22$ in test $\mathrm{j}=1, \ldots, \mathrm{n}=11$ and condition $\mathrm{h}=1,2$. Condition $\mathrm{h}=1$ denotes estimating distance from the boat (to shore), and $\mathrm{h}=2$ denotes estimating distance from shore back to the boat. Associated with these observations are predictor variables:

- $\mathrm{x}_{\mathrm{ijh}}$, the log of the true distances as measured by individual $\mathrm{i}$ in test $\mathrm{j}$ and condition $\mathrm{h}$.

- over $_{i j h}$, a variable that quantifies the error in the previous estimate made by individual $i$. For the first measurement $(\mathrm{j}=1)$, the value over ${ }_{i 1 h}$ is assigned the value 0 . For all other measurements, it is given as

over $_{i j h}=y_{i, j-1, h}-x_{i, j-1, h}$

- $\mathrm{bf}_{\mathrm{i}}$, an indicator variable that denotes whether individual $\mathrm{i}$ first observed from the boat or from shore. If individual i first estimated distances from the boat, $\mathrm{bf}_{\mathrm{i}}$ is assigned the value 1 ; otherwise, $b f_{i}$ is assigned the value 0 .
- expert $_{\text {ih }}$, an indicator variable that denotes whether individual $i$ in condition $h$ is experienced (has already participated in another condition). If individual $i$ in condition $h$ has previously estimated the other condition, expert $\mathrm{ih}_{\mathrm{ih}}$ is assigned the value 1 ; otherwise, expert ${ }_{i h}$ is assigned the value 0 .

The latter three variables are included to determine whether there is evidence that (i) the previous estimate has an effect on the current estimate, (ii) starting in the boat (or shore) affects estimation, or (iii) previously having estimated affects estimation.

We specify a multilevel (or hierarchical) model for the data. The first level of the model is

$$
\begin{aligned}
\mathrm{y}_{\mathrm{ijh}} & \stackrel{\mathrm{ind}}{\sim} \mathrm{N}\left(\beta_{\mathrm{ih}}+\gamma_{\mathrm{ih}} \mathrm{x}_{\mathrm{ijh}}+\phi_{1} \text { over }_{\mathrm{ijh}}+\phi_{2} \mathrm{bf}_{\mathrm{i}}+\phi_{3} \text { expert }_{\mathrm{ih}}, \sigma_{\mathrm{ih}}^{2}\right), \mathrm{i} \\
& =1, \ldots, 22, \mathrm{j}=1, \ldots, \mathrm{n}, \mathrm{h}=1,2,
\end{aligned}
$$

where ind denotes independent random variables and $\mathrm{N}$ $\left(\mu, \sigma^{2}\right)$ denotes a random normal variable with mean $\mu$ and variance $\sigma^{2}$. This model allows the intercept $(\beta)$, slope $(\gamma)$ and error variance $(\sigma)$ to vary by individual and condition.

The second level of the model describes the variability in the individual specific parameters. For both the intercept and slope terms, we consider additive individual $(\chi)$ and condition $(\alpha, \delta)$ effects with common variance:

$$
\begin{array}{lll}
\beta_{\text {ih }} & \stackrel{\text { ind }}{\sim} \mathrm{N}\left(\chi_{\mathrm{i} 1}+\alpha_{\mathrm{h}}, \sigma_{\mathrm{b}}^{2}\right), \mathrm{i}=1, \ldots, 22, & \mathrm{~h}=1, \ldots, 2 \\
\gamma_{\text {ih }} & \stackrel{\text { ind }}{\sim} \mathrm{N}\left(\chi_{\mathrm{i} 2}+\delta_{\mathrm{h}}, \sigma_{\mathrm{g}}^{2}\right), \mathrm{i}=1, \ldots, 22, & \mathrm{~h}=1, \ldots, 2,
\end{array}
$$

where the constraint $\alpha_{1}=\delta_{1}=0$ for identifiability. For the standard deviation terms $\sigma$ we specify

$\log \left(\sigma_{\text {ih }}\right) \stackrel{\text { ind }}{\sim} \mathrm{N}\left(\mu_{\sigma \mathrm{h}}, \sigma_{\sigma \mathrm{h}}^{2}\right), \mathrm{i}=1, \ldots, 22, \quad \mathrm{~h}=1, \ldots, 2$.

The third level of the model describes a model for the individual effects $\chi$ :

$\chi_{\mathrm{ik}} \stackrel{\text { ind }}{\sim} \mathrm{N}\left(\mu_{\mathrm{xk}}, \sigma_{\chi \mathrm{k}}^{2}\right), \mathrm{i}=1, \ldots, 22, \quad \mathrm{k}=1,2$.

We complete the model specification with priors for all remaining parameters. We assign vague or weakly informative independent prior distributions (Gelman et al. 2014). In particular,

$$
\begin{array}{cc}
\alpha_{2} & \sim \mathrm{N}(0,100000) \\
\delta_{2} & \sim \mathrm{N}(0,100000) \\
\mu_{\sigma \mathrm{h}} & \sim \mathrm{N}(0,100), \mathrm{h}=1, \ldots, 2 \\
\sigma_{\mathrm{b}} & \sim \mathrm{t}_{3}(0,25) \mathrm{T}(0, \infty) \\
\sigma_{\mathrm{g}} & \sim \mathrm{t}_{3}(0,25) \mathrm{T}(0, \infty) \\
\sigma_{\sigma \mathrm{h}} & \sim \mathrm{t}_{3}(0,25) \mathrm{T}(0, \infty), \mathrm{h}=1, \ldots, 3 \\
\sigma_{\mu \mathrm{k}} & \sim \mathrm{N}(0,100000), \mathrm{k}=1,2 \\
\sigma_{\mathrm{xk}} & \sim \mathrm{t}_{3}(0,25) \mathrm{T}(0, \infty), \mathrm{k}=1,2,
\end{array}
$$

where $\mathrm{t}_{v}\left(\mu, \sigma^{2}\right)$ denotes a $t$ distribution with $v$ degrees of freedom, location $\mu$ and scale parameter $\sigma$ and $\mathrm{T}(\mathrm{a}, \mathrm{b})$ denotes truncation between values $a$ and $b$. 


\section{References}

Baird, R. W., \& Burkhart, S. M. (2000). Bias and variability in distance estimation on the water: Implications for the management of whale watching. Paper presented at the IWC Meeting Document SC/52/ WW1.

Brenner, E., \& Van Damme, W. J. (1999). Perceived distance, shape and size. Vision Research, 39(5), 975-986.

Daum, S. O., \& Hecht, H. (2009). Distance estimation in vista space. Attention, Perception, \& Psychophysics, 71(5), 1127-1137.

Durgin, F. H., \& Li, Z. (2011). Perceptual scale expansion: An efficient angular coding strategy for locomotor space. Attention, Perception, \& Psychophysics, 73(6), 1856-1870.

Fine, B. J., \& Kobrick, J. L. (1983). Individual differences in distance estimation: Comparison of judgments in the field with those from projected slides of the same scenes. Perceptual and Motor Skills, 57(1), 3-14.

Franklin, S. S., Ross, H. E., \& Weltman, G. (1970). Size-distance invariance in perceptual adaptation. Psychonomic Science, 21(4), 229-231.

Gelman, A., Carlin, J., Stern, H., Dunson, D., Vehtari, A., \& Rubin, D. (2014). Bayesian analysis (3rd ed.). New York: Chapman \& Hall.
Gelman, A., \& Hill, J. (2006). Data analysis using regression and multilevel/hierarchical models. Cambridge: Cambridge University Press.

Gibson, J. J. (1979). The ecological approach to visual perception. Hillsdale: Erlbaum.

Gogel, W. C. (1971). The validity of the size-distance invariance hypothesis with cue reduction. Attention, Perception, \& Psychophysics, 9(1), 92-94.

Higashiyama, A., \& Shimono, K. (1994). How accurate is size and distance perception for very far terrestrial objects? Function and causality. Perception \& Psychophysics, 55(4), 429-442.

Ross, H. E. (1967). Water, fog and the size-distance invariance hypothesis. British Journal of Psychology, 58(3), 301.

Willey, C. R., \& Jackson, R. E. (2014). Visual field dependence as a navigational strategy. Attention, Perception, \& Psychophysics, 76(4), 1036-1044.

Ziemer, C. J., Plumert, J. M., Cremer, J. F., \& Kearney, J. K. (2009). Estimating distance in real and virtual environments: Does order make a difference? Attention, Perception, \& Psychophysics, 71(5), $1095-1106$. 